\title{
Urotensine II et somatostatine : les retrouvailles de deux vieilles cousines
}

Hervé Tostivint, Isabelle Lihrmann, Hubert Vaudry

> L'urotensine II (UII) est un neuropeptide cyclique qui a été initialement isolé à partir de l'urophyse $(\rightarrow)$ des poissons téléostéens en raison de ses propriétés vasomotrices [1]. Par la suite, l'existence de I'UII a été établie chez l'en- $(\rightarrow) \mathrm{m} /$ s1999, n०5, p. 709 semble des vertébrés et notamment chez I'homme [2-4]. Chez les tétrapodes qui, contrairement aux poissons, sont dépourvus d'urophyse, l'Ull est produite presque exclusivement par les motoneurones de la moelle épinière et du tronc cérébral.

L'UII présente d'importantes similitudes de structure avec un autre peptide, la somatostatine (SS), à tel point, d'ailleurs, qu'elle a été initialement décrite comme un somatostatin-like peptide par ses découvreurs [1]. De fait, I'UII et la SS sont des molécules cycliques, de taille similaire, qui possèdent l'une et l'autre le motif Phe-TrpLys dans leur région cyclique (Figure 1). L'hypothèse selon laquelle I'UII et la SS seraient issues d'un même peptide ancestral a donc été proposée [1].

Elle a été toutefois rapidement abandonnée car il est apparu que les précurseurs de I'UII et de la SS, malgré une organisation générale similaire, ne présentaient quasiment aucune identité de séquence [3]. Par la suite, des variants de l'UII et de la SS, appelés respectivement UII-related peptide (URP) et cortistatine (CST) $(\rightarrow)$, ont été découverts [5-7] (Figure 1), confortant l'idée selon laquelle

$(\rightarrow) \mathrm{m} / \mathrm{s}$ ces peptides appartenaient $1996, \mathrm{n}^{\circ} 10$, à deux familles distinctes.

p. 1131

${ }^{1}$ Urophyse : organe neurosécréteur, analogue à la neurohyophyse, situé à l'extrémité caudale de la moelle épinière des poissons téléostéens.
Lorsque les séquences de gènes apparentés sont trop divergentes, les méthodes classiques de la phylogénie moléculaire s'avèrent souvent inefficaces pour reconstituer leur histoire, voire même attester de leur origine commune. II existe cependant un moyen de contourner le problème en examinant leur localisation chromosomique. En effet, lorsqu'un gène se duplique, la position relative des copies qu'il engendre dépend souvent directement du mécanisme de duplication qu'il a subi et peut donc, en retour, témoigner de la réalité de l'événement. La localisation des gènes sur leurs chromosomes est devenue une tâche relativement aisée, grâce aux données de séquençage et de cartographie accumulées chez de nombreuses espèces. Appliquées au cas de l'Ull et de la SS, elles permettent aujourd'hui de proposer de nouvelles hypothèses sur l'origine de ces deux familles de neuropeptides [8].
Inserm U413,

Laboratoire de Neuroendocrinologie Cellulaire

et Moléculaire, Institut Fédératif

de Recherches Multidisciplinaires

sur les Peptides (IFRMP23),

Université de Rouen,

76821 Mont-Saint-Aignan, France.

herve.tostivint@univ-rouen.fr

hubert.vaudry@univ-rouen.

La première ébauche de la séquence du génome humain a très vite permis de constater que les gènes de l'Ull et de la CST sont situés au voisinage l'un de l'autre à l'extrémité du bras court du chromosome 1, en lp36 (Figure 1) [8]. A l'époque toutefois, le gène de l'URP n'était pas encore connu, et la proximité des deux gènes pouvait être considérée comme fortuite. Cette hypothèse s'est néanmoins trouvée rapidement invalidée lorsque le gène de l'URP a été découvert, car sa localisation, en $3 q 28$, s'est avérée extrêmement proche de celle du gène de la SS (Figure 1) [5]. Par la suite, il a été établi que les positions relatives de ces quatre gènes sont parfaitement con-

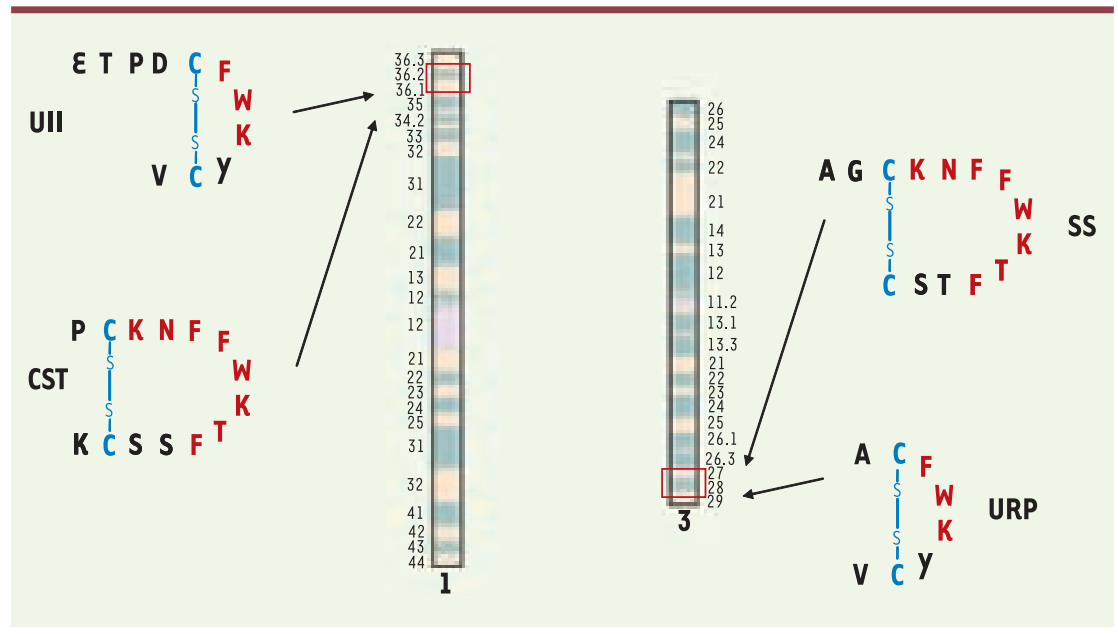

Figure 1. Comparaison de la structure des peptides apparentés à l'urotensine II (UII) et à la somatostatine (SS), ainsi que de la localisation de leurs gènes. CST : coristatine ; URP : urotensin II-related peptide. 
servées chez plusieurs autres espèces, notamment chez le poulet et le poisson zèbre [8].

De ces observations, il ressort que les gènes de la SS et de la CST, d'une part, et ceux de l'UII et de l'URP, d'autre part, ont été très probablement engendrés par duplication segmentaire (Figure 2). À l'appui de cette thèse, nous avons identifié chez l'homme plusieurs autres paires de gènes dupliqués, dont l'une des copies est localisée en $1 p 36$ et l'autre en $3 q 28$, démontrant ainsi que ces deux régions sont paralogues [8].

La localisation actuelle des gènes de I'UII et de I'URP, et de ceux de la SS et de la CST, suggère également que les deux gènes ancestraux dont ils dérivent étaient eux-mêmes vraisemblablement très proches l'un de l'autre, sur le même segment de chromosome. Il est peu probable que cette disposition soit le simple fait du hasard. Compte tenu des similitudes de structure entre les deux peptides évoquées précédemment, il est légitime d'attribuer la proximité de leur gène au résultat d'une duplication en tandem (Figure 2). Certes, les gènes de l'Ull et de la CST, de même

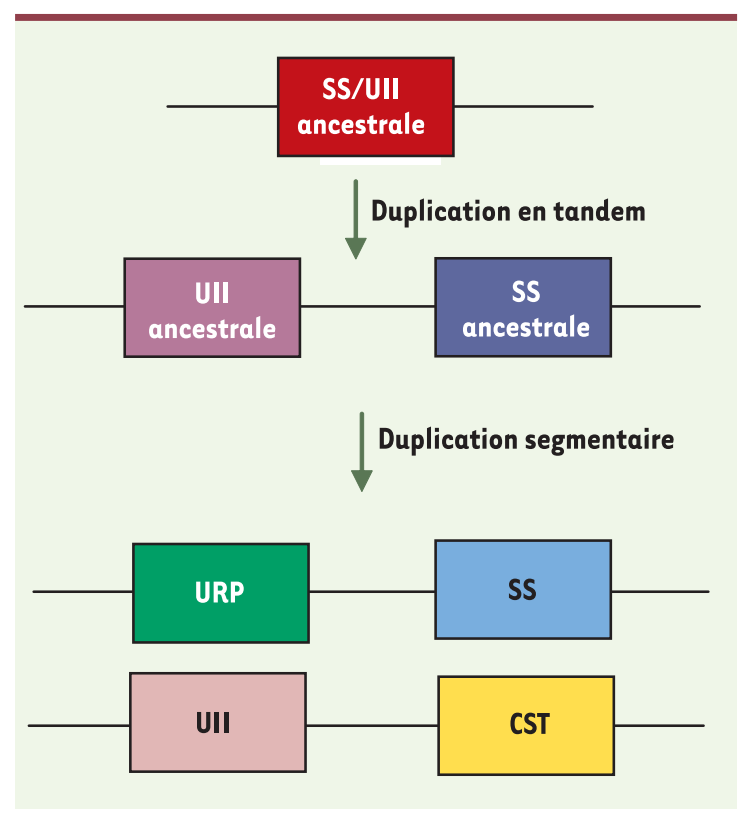

Figure 2. Modèle illustrant les processus évolutifs à l'origine des gènes de la famille de l'urotensine II (UII) et de la somatostatine (SS). CST : cortistatine ; URP : urotensin II-related peptide. que ceux de la SS et de I'URP ne sont pas strictement disposés en tandem puisqu'ils sont séparés par plusieurs autres gènes sur une distance qui varie de 1,1 à 3,7 Mb suivant les espèces étudiées. Néanmoins, on peut parfaitement imaginer qu'ils ont été engendrés dans cette configuration en tandem puis ont été ensuite séparés par insertion de nouveaux gènes. Une autre possibilité serait que la duplication originelle n'ait pas affecté seulement le gène ancestral putatif de l'Ull et de la SS mais tout un groupe d'autres gènes situés dans son voisinage. A l'appui de cette dernière hypothèse, nous avons identifié chez l'homme plusieurs autres paires de gènes en $1 p 36$. Ils semblent donc avoir été dupliqués ensemble [8]. Quelle que soit l'hypothèse exacte, l'ensemble de ces observations plaide clairement en faveur d'une origine commune des gènes de l'Ull et de la SS (Figure 2).

Il est par ailleurs intéressant de noter que la parenté des peptides de la famille de I'UII et de la SS peut être mise en parallèle avec celle de leurs récepteurs. En effet, le récepteur de I'UII (UTR) [4] et ceux de la SS, dont on connaît actuel-

lement 5 sous-types (sstl-5) [9], appartiennent à la même famille des récepteurs à sept domaines transmembranaires couplés aux protéines $G$ et partagent un taux de similarité relativement important. Or, chez l'homme, nous avons constaté que les gènes UTR et sst2 sont localisés dans la même région du chromosome 17, suggérant que les deux gènes proviennent également d'une duplication en tandem. Cette observation est en accord avec l'idée d'une co-évolution des gènes de la famille de I'UII/SS et des gènes codant leurs récepteurs.
Le moment exact où les gènes de I'UII et la SS sont apparus, puis à leur tour se sont dupliqués, est difficile à préciser. On peut cependant penser que la première duplication est antérieure à l'émergence des lamproies, car l'Ull et la SS existent déjà dans ce groupe, alors que seule la SS est présente chez les myxines. Rappelons que les lamproies et les mixines, en tant que représentants du groupe des agnathes, sont considérées comme des descendants des vertébrés les plus primitifs, apparus il y a plus de 550 millions d'années. La seconde duplication, quant à elle, serait intervenue avant l'apparition des poissons osseux, puisque des séquences apparentées à I'URP et à la CST sont détectées à la fois chez le poisson-zèbre, le Fugu et le Tetraodon. Cette seconde duplication pourrait coïncider avec l'un des deux événements de tétraploïdisation qui est vraisemblablement intervenu chez les vertébrés, il y a $450 \mathrm{~mA}$ environ, et qui serait responsable de la diversification de nombreuses autres familles de neuropeptides [10].

À ce jour, ni l'UII ni la SS n'ont été identifiées chez les invertébrés. Pourtant, chez la drosophile, une étude récente a établi l'existence d'un gène apparenté à ceux codant les récepteurs de la SS, dont l'environnement chromosomique est homologue à celui des gènes sst 3 et $s s t 5$ humains [11]. De façon intéressante, le récepteur codé par ce gène a pour ligand naturel un peptide cyclique, comme la SS, mais qui ne présente aucune autre similarité de structure avec elle [12]. La question actuellement non résolue est de savoir si ce peptide, qui appartient à la famille des allatostatines, est ou non apparenté à la SS. D'un point de vue fonctionnel, il est troublant de constater que, comme la SS, il est exprimé dans des cellules neuroendocrines et participe au contrôle de la croissance comme inhibiteur de sécrétions hormonales, en premier lieu, celle de l'hormone juvénile par les corps allates, d'où sa famille tire son nom. $\diamond$ Urotensin II and somatostatin : two old cousins get together again 


\section{RÉFÉRENCES}

1. Pearson D, Shively JE, Clark BR, et al. Urotensin II : a somatostatin-like peptide in the caudal neurosecretory system of fishes. Proc Natl Acad Sci USA $1980 ; 77: 5021-4$

2. Coulouarn $Y$, Lihrmann I, Jégou $S$, et al. Cloning of the cDNA encoding the urotensin II precursor in frog and human reveals intense expression of the urotensin II gene in motoneurons of the spinal cord. Proc Natl Acad Sci USA 1998; 95 : 15803-8.

3. Conlon JM, Tostivint H, Vaudry H. Somatostatin- and urotensin II-related peptides : molecular diversity and evolutionary perspectives. Regul Pept 1997; 69: 95-103.

4. Lihrmann I, Bern HA, Vaudry H. Urotensin II and urotensin II-related peptide. In : Kastin AJ, ed.
Handbok of biologically active peptides. Paris : Elsevier, 2006 ; 109 : 815-23.

5. Sugo T, Murakami $Y$, Shimomura $Y$, et al. Identification of urotensin II-related peptide as the urotensin IIimmunoreactive molecule in the rat brain. Biochem Biophys Res Commun $2003 ; 310: 860-8$.

6. Tostivint H, Lihrmann I, Bucharles C, et al. Occurrence of two somatostatin variants in the frog brain: characterization of the cDNAs, distribution of the mRNAs, and receptor-binding affinities of the peptides. Proc Natl Acad Sci USA 1996; 93 : 12605-10.

7. de Lecea L, Criado JR, Prospero-Garcia 0, et al. A cortical neuropeptide with neuronal depressant and sleep-modulating properties. Nature 1996 ; $381: 242-5$.

8. Tostivint $\mathrm{H}$, Joly L, Lihmann I, et al. Comparative genomics provides evidence for close evolutionary relationships between the urotensin II and somatostatin gene families. Proc Natl Acad Sci USA $2006 ; 103: 2237-42$.

9. Olias G, Viollet C, Kusserow H, et al. Regulation and function of somatostatin receptors. J Neurochem $2004 ; 89$ : 1057-91.

10. Conlon JM, Larhammar. The evolution of neuroendocrine peptides. Gen Comp Endocrinol 2005 $142: 53-9$.

11. Popovici C, Leveugle M, Birnbaum D, Coulier F. Coparalogy: physical and functional clusterings in the human genome. Biochem Biophys Res Commun 2001 ; $288: 362-70$.

12. Kreienkamp HJ, Larusson HJ, Witte I, et al. Functional annotation of two orphan $\mathrm{G}$-coupled receptors, Drostarl and -2, from Drosophola melanogaster and their ligands by reverse pharmacology. J Biol Chem $2002 ; 277: 39937-43$

\section{NOUVELlE}

\section{Nouvelles stratégies d'obtention des cellules souches embryonnaires}

Philippe Taupin

\author{
National Neuroscience Institute, Singapour. \\ National University of Singapour. \\ Nanyang Technological University, Singapour. \\ 11Jalan Tan Tock Seng, 308433 Singapour. \\ obgpjt@nus.edu.sg
}

$>$ Les cellules souches embryonnaires (CSE) sont des cellules indifférenciées, qui prolifèrent in vitro, et ont le potentiel de se différencier dans tous les types cellulaires de l'organisme, suscitant, à ce titre, un intérêt thérapeutique. Les CSE sont dérivées de la masse cellulaire interne de blastocystes, nécessitant la destruction de ces derniers [1]. De ce fait, l'utilisation de lignées de CSE humaines existantes, et la production de nouvelles lignées chez l'homme sont soumises à une réglementation stricte $(\rightarrow)$ et se heurtent à certaines oppositions. Des études récentes tentent de $(\rightarrow)$ $\mathrm{m} / \mathrm{s} 2003$, n० 6-7, p. 683 répondre à ces limitations en proposant des stratégies d'obtention de CSE sans destruction des embryons.

$y$. Chung et al. rapportent l'établissement, chez la souris, de lignées de CSE à partir de blastomères - stade du développement où l'œuf ne comporte que 8 cellules - sans affecter la poursuite du développement des embryons [2]. Les lignées sont établies à partir d'un seul blastomère, tandis que les embryons ne comportant plus que 7 cellules se développent normalement après implantation dans l'utérus. L'établissement de ces lignées nécessite l'agrégation des blastomères avec des CSE préexistantes. Ces dernières sont marquées avec la GFP (green fluorescent protein), ce qui permet de les distinguer des cellules issues du blastomère (exprimant lacz). En l'absence d'un tel support cellulaire, les blastomères ne se divisent pas et se différencient. Après 2 à 4 jours en culture, les cellules dérivées de blastomères (non marquées à la GFP) se sont divisées et sont alors séparées des CSE fluorescentes, et cultivées selon les mêmes protocoles que les CSE. L'absence de contamination par les CSE «nourricières » est attestée par l'absence de transcrits codant pour la GFP, et par l'absence de fusion cellulaire. Les auteurs ont ainsi établi cinq lignées cellulaires de CSE qui possèdent les caractéristiques habituelles: pluripotentialité et capacité à former des tératomes chez la souris. En particulier, après injection dans des blastocystes, elles participent à la formation de tous les tissus chez l'hôte, y compris les lignées germinales. Elles expriment également des marqueurs des CSE (0ct-4, SSEA-1). Des lignées trophoblastiques ont pu également être établies en cultivant les CSE en présence d'un facteur trophique, le FGF-4. Ces résultats démontrent que des cellules isolées de blastomères peuvent être utilisées pour dériver des lignées de CSE, une approche qui n'interfère pas avec le potentiel de développement de l'embryon. L'efficacité est cependant faible, puisque seules 12 lignées de CSE ont été dérivées à partir de 125 blastomères, alors que le taux d'efficacité est de $25 \%-30 \%$ à partir de blastocystes. Dans le cas du transfert nucléaire, et sans revenir sur le scandale récent de l'équipe coréénne dont la revue s'est faite l'écho $\rightarrow$, certaines des réticences et craintes concernaient la «création » artificielle d'un «embryon», s2006, $n^{\circ} 2$, p. 218 